\title{
The microbiology of bacterial peritonitis due to appendicitis in children
}

\author{
O. Obinwa $\cdot$ M. Casidy $\cdot$ J. Flynn
}

Received: 3 August 2013/Accepted: 3 December 2013/Published online: 18 December 2013

(C) The Author(s) 2013. This article is published with open access at Springerlink.com

\begin{abstract}
Aim The aim of this study was to investigate the microbiology of secondary bacterial peritonitis due to appendicitis and the appropriateness of current antimicrobial practice in one institution.

Methods A 14-year retrospective single-centre study of 69 consecutive paediatric patients (age 1-14 years) with appendicitis-related peritonitis and positive peritoneal specimen cultures was conducted. Post-operative outcomes, microbiology and antibiotic susceptibility of peritoneal isolates were analysed in all patients.

Results Escherichia coli was identified in 56/69 (81\%) peritoneal specimens; four isolates were resistant to amoxicillin-clavulanate, and one other isolate was resistant to gentamicin. Anaerobes were identified in 37/69 (54\%) peritoneal specimens; two anaerobic isolates were resistant to amoxicillin-clavulanate and one isolate was resistant to metronidazole. Pseudomonas aeruginosa was identified in 4/69 (6\%) peritoneal specimens, and all were susceptible to gentamicin. Streptococcal species (two Group F streptococci and three $\beta$-haemolytic streptococci) were identified in 5/69 (7\%) specimens, and all were susceptible to amoxicillin-clavulanate. Combination therapy involving amoxicillin-clavulanate and aminoglycoside is appropriate empirical treatment in 68/69 (99\%) patients. Addition of metronidazole to this regime would provide $100 \%$ initial empirical coverage. Inadequate initial empiric antibiotic
\end{abstract}

O. Obinwa $(\bowtie) \cdot$ J. Flynn

Department of Surgery, Portiuncula Hospital, Ballinasloe,

Ireland

e-mail: obinnaobinwa@rcsi.ie

M. Casidy

Department of Pathology, Portiuncula Hospital, Ballinasloe, Ireland treatment and the presence of amoxicillin-clavulanate resistant $E$. coli were independent predictors of the postoperative infectious complications observed in 14/69 (20\%) patients.

Conclusion E. coli and mixed anaerobes are the predominant organisms identified in secondary peritonitis from appendicitis in children. Inadequate initial empirical antibiotic and amoxicillin-clavulanate resistant E. coli may contribute to increased post-operative infectious complications. This study provides evidence-based information on choice of combination therapy for paediatric appendicitisrelated bacterial peritonitis.

Keywords Paediatric C Children - Appendicitis · Peritonitis - Antibiotic susceptibility

\section{Introduction}

Peritonitis is an inflammation of the membrane lining the inside of the abdomen/pelvis and all of the internal organs. Secondary peritonitis, as opposed to primary peritonitis, which occurs spontaneously, is the result of some other disease process [1]. In children, the most common cause of secondary peritonitis is perforated appendicitis and intraabdominal abscess arising from acute appendicitis [1]. Secondary peritonitis in children is usually community acquired and accounts for prolonged hospitalisation $[1,2]$. The aetiology of this disease is predominantly microbial with organisms from gut flora namely Enterobacteriaceae (coliforms) and anaerobes as pathogens [1, 3, 4].

Effective antimicrobials currently in use in Europe and throughout the world are fast losing ground as these causative pathogens, particularly the Enterobacteriaceae, acquire resistance to newly introduced antibiotics [5]. 
Despite this awareness, studies of secondary peritonitis due to appendicitis in children are limited [1, 6-9]. Treatment protocols vary widely and are guided by anti-microbial resistance patterns [7, 10, 11]. Complete antimicrobial coverage may be achieved using multiple agents $[7,10$, 12-16]. However, targeted antibiotic treatment is preferable in the interest of decreasing resistance [1, 17-19].

Currently, in Ireland, antibiotic monotherapy (usually amoxicillin-clavulanate) is recommended for use in the management of uncomplicated appendicitis. For peritonitis, the recommendation is the use of combination antibiotic therapy involving amoxicillin-clavulanate and an aminoglycoside, gentamicin. In penicillin-sensitive patients, a combination of gentamicin or cephalosporin and metronidazole may be effective. The aim of this study was to investigate the microbiology of secondary bacterial peritonitis due to appendicitis and the appropriateness of current antimicrobial practice in one institution.

\section{Methods}

A retrospective review of consecutive children (age between 1 and 14 years) presenting with secondary bacterial peritonitis due to appendicitis between January 1995 and December 2008 was conducted in one institution.

\section{Patients}

There were 105 children with macroscopic findings of perforated appendicitis or abscess during appendicectomy during the study period. 25 (24\%) of these were excluded because no fluid specimens were sent for microbiological analysis and $11(10 \%)$ were further excluded because their peritoneal fluid specimens did not grow any organism. The remaining 69 children with perforated appendicitis and intra-abdominal abscess and who had positive cultures formed the principal cohort for analysis. It is important to note that subjects with simple acute non-perforated appendicitis or gangrenous appendicitis (macroscopic) without evidence of perforation were not part of this study.

\section{Specimen culture}

Peritoneal fluid specimens in the cohort were sent directly to the laboratory or kept at $4{ }^{\circ} \mathrm{C}$ until the next day if they were collected after hours. For aerobic culture, the fluid specimens were inoculated onto Columbia blood agar and MacConkey agar without salt. The plates were incubated at $37^{\circ} \mathrm{C}$ in air atmosphere and were examined 24 and $48 \mathrm{~h}$ after incubation.

For anaerobic culture, the fluid specimens were plated onto Columbia blood agar, neomycin blood agar, and nalidixic acid agar and each plated agar further impregnated with metronidazole discs so as to guide sensitivity analysis. All plates were incubated in an anaerobic gas jar with $\mathrm{O}_{2}$ levels $<1 \%$ and $\mathrm{CO}_{2}$ levels between 9 and $13 \%$ and examined for growth at 24, 48, 96 and $120 \mathrm{~h}$ after incubation.

All aerobic isolates were fully identified. Specimens with anaerobic isolates having more than one anaerobe identified were classified as mixed anaerobe. Sensitivity analysis was conducted with the aid of a rapid and automated VITEC-2 compact system (Biomérieux, France). This system has been in place since 2005. The Clinical and Laboratory Standards Institute dilution method was used for sensitivity testing between 1998 and 2005, and before this time, the Stokes' method of sensitivity testing was used.

\section{Data collection}

Data recorded included: demographic data, microbiological data (peritoneal fluid specimens and susceptibility to antibiotics), antibiotic management (initial therapy, changes in therapy, and duration of treatment) and outcomes. Infectious complications were defined as those occurring within 30 days of surgery and included intra-abdominal abscess and wound infection. The intra-abdominal abscesses were confirmed by imaging and microbiological samples. Wound infection was confirmed clinically and by microbiological samples. Patients who received oral doses of antibiotics in the community within a 1-week period before hospital admission were recorded. Adequate empirical antibiotic treatment was defined as resolution of disease with initial or step-down antibiotic treatment after primary surgery. Empirical antibiotic treatment was inadequate if the infection was nonresolving and additional antibiotics were commenced postoperatively based on intraperitoneal fluid culture results.

\section{Data analysis}

Statistical analysis was conducted using the STATA 11.0 (Stata, College Station, TX) software. Continuous data were expressed as median and percentiles (25-75\%) and analysed by a Mann-Whitney $U$ test. Data expressed by percentage of children were analysed by Chi-square and Fisher's exact test as appropriate. Differences were considered statistically significant at the $5 \%$ level $(P<0.05)$. For subgroup analysis, data was classified into two periods: 1995-2002 (36 patients) and 2003-2008 (33 patients). This division was necessary to identify trends, if any, in the use of antibiotics.

Like previous studies, potential variables that may be associated with a higher risk of post-operative infection and hospitalisation in the cohort of patients with appendicitis-related community acquired peritonitis were examined $[1,2]$. Significant risk factors identified after 
univariate testing (defined by $P<0.2$ ) were further tested in a multivariate logistic regression model [1, 2]. Variables associated with $P<0.05$ after multivariate analysis were considered independent factors of risk. Odds ratios and their $95 \%$ confidence intervals were calculated.

\section{Results}

Patients

The 69 children with secondary appendicitis-related bacterial peritonitis included 31 females. The median age at the time of surgery was $8(5-11)$ years.

Clinical outcome

$56 / 69(81 \%)$ patients had localised peritonitis and the remaining 13/69 (19\%) had generalised peritonitis. 68/69 (98.5\%) patients had open appendicectomy. Two children underwent a 'second look' operation in both cases for prolonged ileus in the post-operative period. 14/69 (20\%) patients had infective complications. There were 18 infective complications recorded in the 14 patients ( 9 intraabdominal collections, 5 superficial wound infections, 2 deep wound infections/dehiscence and 2 chest infections). The median length of stay in hospital was 6 (5-8) days.

\section{Microbiology}

The microorganisms identified in the peritoneal specimens of the 69 patients are shown in Table 1. Single isolates were identified in 31/69 patients (45\%), and multiple isolates were identified in the other $38(55 \%)$ patients. Escherichia coli was identified in 56/69 (81 \%) specimens; Four of these isolates were resistant to amoxicillin-clavulanate and only one of the $E$. coli strains was resistant to the aminoglycoside, gentamicin. There was no isolates of extended spectrum beta lactamases or carbapenem-resistant Enterobacteriaceae identified in any of the specimens, but these may be important in the future. Streptococcal species (two Group F streptococci and three $\beta$-haemolytic streptococci) were identified in 5/69 (7\%) specimens. All the streptococcal species were sensitive to amoxicillinclavulanate. Pseudomonas aeruginosa was identified in 4/69 (6\%) specimens; all were sensitive to gentamicin. Mixed anaerobes were identified in 37/69 (54\%) specimens; two anaerobic isolates were resistant to amoxicillinclavulanate and one isolate was resistant to metronidazole. A resistant-sensitive "synergism" was found between metronidazole and gentamicin for anaerobes and coliforms. Simply put, we noted sensitivity to gentamicin in one case of metronidazole-resistant anaerobes and sensitivity to
Table 1 Microorganisms isolated from peritoneal fluid specimens $(n=69)$

\begin{tabular}{lc}
\hline Microorganisms & $N(\%)$ \\
\hline Aerobes & \\
Gram negative & $56(81)$ \\
$\quad$ Escherichia coli & $5(7)$ \\
Other Enterobacteriaceae & $4(6)$ \\
Pseudomonas aeruginosa & \\
Gram positive & $2(3)$ \\
Group F streptococci & $3(4)$ \\
B-haemolytic streptococci & $37(54)$ \\
Anaerobes &
\end{tabular}

metronidazole was documented in one case of gentamicinresistant coliform.

Antibiotic treatment

All patients received antibiotics pre-operatively. Median duration of intravenous antibiotic treatment was 4 (3-6) days. 9/69 (13\%) patients had received oral antibiotics in the community prior to presentation to hospital (Amoxicillin in six cases, amoxicillin-clavulanate in two cases and erythromycin in one case). For initial in-hospital treatment, 25/69 (36\%) patients received triple drug therapy (amoxicillin-clavulanate/cephalosporin + metronidazole + aminoglycoside) and the other 44/69 (64\%) patients received a double combination of amoxicillin-clavulanate/cephalosporin and metronidazole. We were unable to assess exactly what guided these decisions. Antibiotic treatment changed based on findings at operation without peritoneal specimen culture and sensitivity results in 10/69 (14\%) children. Antibiotic treatment was considered inadequate and modified in accordance with culture results in 4/69 (6\%) children. 13/69 (19\%) patients had de-escalation of treatment following laboratory susceptibility results.

Use of cephalosporin versus amoxicillin-clavulanate combination therapy

Over time, cephalosporin combination therapy became less frequently used as initial treatment in the treatment of secondary community acquired peritonitis. They accounted for $86 \%$ of the empirical treatment from 1995 to 2002 and $67 \%$ of empirical treatment from 2003 to 2008. Amoxicillinclavulanate combination therapy became increasingly used in the treatment of appendicitis-related peritonitis as the use of cephalosporins declined. In 12 out of the 13 patients who had de-escalation of treatment following laboratory susceptibility results, this involved stopping a cephalosporin and commencing amoxicillin-clavulanate instead. 
Table 2 Univariate analysis of factors associated with a risk of postoperative infection in children with secondary peritonitis from appendicitis

\begin{tabular}{|c|c|c|c|}
\hline & $\begin{array}{l}\text { Post-op } \\
\text { infection } \\
(N=14)\end{array}$ & $\begin{array}{l}\text { No post-op } \\
\text { infection } \\
(N=55)\end{array}$ & $P$ \\
\hline Age (years), median (25th-75th) & $\begin{array}{l}8.5 \\
(5-12)\end{array}$ & $8(5-11)$ & 0.512 \\
\hline Sex ratio $\mathrm{F} / \mathrm{M}, n$ & $4 / 10$ & $27 / 28$ & $0.168 *$ \\
\hline Generalised peritonitis, $n(\%)$ & $3(21)$ & $10(18)$ & 0.781 \\
\hline Intra-abdominal abscess, $n(\%)$ & $6(43)$ & $14(25)$ & $0.200 *$ \\
\hline $\begin{array}{l}\text { Use of cefuroxime-metronidazole, } \\
n(\%)\end{array}$ & $9(64)$ & $36(65)$ & 0.935 \\
\hline $\begin{array}{l}\text { Use of amoxicillin-clavulanate } \\
\text { combination, } n(\%)\end{array}$ & $4(29)$ & $13(24)$ & 0.702 \\
\hline \multicolumn{4}{|l|}{ Bacteriology, $n(\%)$} \\
\hline Monomicrobial & $6(43)$ & $27(49)$ & 0.677 \\
\hline Polymicrobial & $8(57)$ & $28(51)$ & \\
\hline Escherichia coli, $n(\%)$ & $11(79)$ & $45(82)$ & 0.781 \\
\hline Anaerobic microorganisms, $n(\%)$ & $8(57)$ & $29(52)$ & 0.767 \\
\hline Pseudomonas aeruginosa, $n(\%)$ & $2(14)$ & $2(3)$ & $0.128 *$ \\
\hline Other Enterobacteriaceae, $n(\%)$ & $2(14)$ & $3(5)$ & 0.255 \\
\hline $\begin{array}{l}\text { E. coli strain resistant to } \\
\text { amoxicillin-clavulanate, } n(\%)\end{array}$ & $3(21)$ & $1(2)$ & $0.005^{*}$ \\
\hline $\begin{array}{l}\text { Streptococci spp (two Group } \\
\mathrm{F}+\text { three } \beta \text {-haemolytic } \\
\text { streptococci), } n(\%)\end{array}$ & $0(0)$ & $5(9)$ & 0.241 \\
\hline Pre-hospital antibiotic treatment & $7(13)$ & $2(14)$ & 0.872 \\
\hline Inadequate initial treatment, $n(\%)$ & $3(21)$ & $1(2)$ & $0.005^{*}$ \\
\hline
\end{tabular}

Comparison by $X^{2}$, Fisher exact test, Mann and Whitney test as appropriate

* Variables with a $P$ value $<0.2$ were tested in a multivariate logistic regression model

The antibiotic susceptibility data suggested that combination therapy involving amoxicillin-clavulanate and gentamicin would have been appropriate empirical treatment in 68/69 (99\%) patients. Addition of metronidazole to this regime would have provided $100 \%$ initial empirical coverage.

Risk factors for post-operative infection and hospital stay

Significant variables associated with increased risk of postoperative infection are presented in Table 2. Results of testing of variables that may be associated with prolonged hospitalisation are presented in Table 3. Independent risk factors for post-operative infectious complications identified on multivariate analysis were: isolation of E. coli resistant to amoxicillin-clavulanate in the peritoneal fluid specimen (OR, 21.88 [1.7-277.2]; $P=0.017$ ) and inadequate initial antibiotic therapy (OR, 18.37 [1.1-321.0]; $P=0.046$ ). Female gender (OR, 3.11 [0.7-14.4]; $P=$ 0.146), isolation of $P$. aeruginosa (OR, 1.55 [0.1-2.2];
Table 3 Univariate analysis of factors associated with a risk of hospitalisation length of stay above 7 days in children with secondary peritonitis from appendicitis

\begin{tabular}{|c|c|c|c|}
\hline & $\begin{array}{l}>7 \text { days } \\
(n=22)\end{array}$ & $\begin{array}{l}<7 \text { days } \\
(n=47)\end{array}$ & $P$ \\
\hline Age (years), median (25th-75th) & $8(4-10)$ & $9(5-11)$ & 0.442 \\
\hline Sex ratio $\mathrm{F} / \mathrm{M}, n$ & $7 / 15$ & $24 / 23$ & $0.134 *$ \\
\hline Generalised peritonitis, $n(\%)$ & $6(27)$ & $7(15)$ & 0.220 \\
\hline Intra-abdominal abscess, $n(\%)$ & $5(23)$ & $15(32)$ & 0.433 \\
\hline $\begin{array}{l}\text { Use of cefuroxime-metronidazole, } \\
n(\%)\end{array}$ & $15(68)$ & $30(64)$ & 0.724 \\
\hline $\begin{array}{l}\text { Use of amoxicillin-clavulanate } \\
\text { combination, } n(\%)\end{array}$ & $6(27)$ & $11(23)$ & 0.728 \\
\hline \multicolumn{4}{|l|}{ Bacteriology, $n(\%)$} \\
\hline Monomicrobial & $7(32)$ & $21(45)$ & $0.069 *$ \\
\hline Polymicrobial & $15(68)$ & $26(55)$ & \\
\hline Escherichia coli, $n(\%)$ & $19(86)$ & $37(79)$ & 0.449 \\
\hline Anaerobic microorganisms, $n(\%)$ & $15(68)$ & $22(47)$ & $0.097 *$ \\
\hline Pseudomonas aeruginosa, $n(\%)$ & $1(5)$ & $3(6)$ & 0.761 \\
\hline Other Enterobacteriaceae, $n(\%)$ & $2(9)$ & $3(6)$ & 0.686 \\
\hline $\begin{array}{l}\text { E.coli strain resistant to } \\
\text { amoxicillin-clavulanate, } n(\%)\end{array}$ & $2(9)$ & $2(4)$ & 0.423 \\
\hline $\begin{array}{l}\text { Streptococci spp (two Group } \\
\mathrm{F}+\text { three } \beta \text {-haemolytic } \\
\text { streptococci), } n(\%)\end{array}$ & $0(0)$ & $5(11)$ & $0.112 *$ \\
\hline Pre-hospital antibiotic treatment & $7(15)$ & $2(9)$ & 0.505 \\
\hline Inadequate initial treatment, $n(\%)$ & $2(9)$ & $2(4)$ & 0.423 \\
\hline $\begin{array}{l}\text { Length of intravenous antibiotic } \\
\text { treatment, median }(25 \text { th }-75 \text { th })^{\mathrm{a}}\end{array}$ & $\begin{array}{l}5.5 \\
(4.7-7)\end{array}$ & $4(3-4.7)$ & $0.001 *$ \\
\hline
\end{tabular}

Comparison by $X^{2}$, Fisher exact test, Mann and Whitney test as appropriate

* Variables with a $P$ value $<0.2$ were tested in a multivariate logistic regression model

${ }^{\text {a }}$ Included variable is a surrogate for disease severity and not a risk factor per se

$P=0.7697)$ or finding of appendicular abscess (OR, 2.68 [0.6-11.8]; $P=0.193$ ) did not reach statistical significance on multivariate analysis.

We did not identify any significant risk factor for prolonged hospitalisation more than 7 days in patients with appendicitisrelated peritonitis on multivariate analysis. We, however, found that the duration of hospitalisation was directly related to the duration of intravenous treatment required to treat each case based on the severity and clinical response.

\section{Discussion}

In the peritoneal samples of 69 children with secondary peritonitis from appendicitis, E. coli was identified in 56/69 $(81 \%)$ peritoneal specimens; four isolates were resistant to amoxicillin-clavulanate, and one other isolate was resistant to gentamicin. Anaerobes were identified in 37/69 (54\%) 
peritoneal specimens; two anaerobic isolates were resistant to amoxicillin-clavulanate and one isolate was resistant to metronidazole. $P$. aeruginosa was identified in 4/69 (6\%) peritoneal specimens, and all were susceptible to gentamicin. Streptococcal species (two Group F streptococci and three $\beta$-haemolytic streptococci) were identified in 5/69 (7\%) specimens, and all were susceptible to amoxicillinclavulanate. Post-operative infectious complications occurred in 14/69 (20\%) patients and predictors of increased post-operative complications were identified. The rationale for use of combination therapy in the treatment of secondary peritonitis was also presented.

The current study may differ from previous studies due to the homogeneity of the group studied but striking other similarities need to be underpinned. E. coli and Bacteroides fragilis (anaerobe) are the main pathogens involved in paediatric appendicitis-related peritonitis [1, 8, 9, 20]. Isolation of $E$. coli resistant to amoxicillin-clavulanate may be associated with post-operative peritonitis [1]. Appropriate initial antimicrobial therapy may predict successful treatment of peritonitis [21]. Isolation of $P$. aeruginosa in peritoneal specimens may be associated with post-appendicectomy surgical infections in the absence of appropriate primary antibiotics [4, 22].

Dumont et al. [1] evaluated microbiology and antimicrobial susceptibility of peritoneal isolates in children who underwent surgery for community acquired peritonitis in a single surgical centre. The study's sample size was similar to that of this study and included only 70 patients: 69 children with peritonitis from appendicitis and 1 from perforation of the small intestine. They found that E. coli and anaerobes were the main pathogens involved in paediatric community acquired peritonitis. They found a $10.4 \%$ resistance rate of coliforms to amoxicillin-clavulanate. Similar to the current study, they showed that the presence of E. coli resistant to amoxicillin-clavulanate was an independent risk factor associated with post-operative peritonitis.

Krobot et al. [21], in a multicentre study of 162 patients with perforated appendicitis, found that appropriateness of initial parenteral antibiotic therapy was a predictor of clinical success and length of stay. Similarly, they demonstrated a high risk of post-operative infections in patients with inadequate empirical treatment.

Two studies in the paediatric population had found a positive correlation between isolation of $P$. aeruginosa in peritoneal specimens and post-appendicectomy surgical infections [4, 22]. Chen et al. [4] isolated $P$. aeruginosa in $18 / 117(15 \%)$ fluid specimens of patients with appendicitis and demonstrated a positive correlation between isolation of $P$. aeruginosa and surgical site infections. They found that $P$. aeruginosa was frequently not covered by chosen prophylactic antibiotics. $7 / 18(39 \%) P$. aeruginosa in that study was resistant to cefuroxime, and they identified pseudomonas in peritoneal specimens of 5/8 (63\%) patients who later developed surgical site infections. Yellin et al. [22], also reported a high rate of infectious complications in patients with appendicitis from whom $P$. aeruginosa were isolated. Compared to these two studies, this study identified $P$. aeruginosa in 4/69 (6\%) specimens and all 4 isolates were sensitive to gentamicin. We found that the four patients with $P$. aeruginosa in their peritoneal specimens had been on cefuroxime and metronidazole as empirical treatment; two of these patients subsequently developed significant intra-abdominal infection and required switch to piperacillin-tazobactam and gentamicin following drainage procedures.

Conflicting data with respect to the role of $P$. aeruginosa in the outcome of peritonitis may be explained by the lack of appropriate antibiotics in the primary treatment protocols. The summary of the data is that addition of an aminoglycoside is paramount when considering treatment for appendicitis-related peritonitis, and that inadequate initial empirical treatment may lead to post-operative infectious complications [1, 23]. Pseudomonas species are also inherently resistant to amoxicillin-clavulanate, and postoperative infections might develop if this antibiotic were to be used alone in the treatment of associated peritonitis.

Knowing the microbial and antibiotic resistance profile is critical in an attempt to provide the best empirical antibiotic treatment for secondary peritonitis arising from appendicitis in children [1]. There is no single empirical antibiotic known to reduce post-appendicectomy infectious complications in patients with complicated appendicitis [3, $8,14,21,24]$. The current local policies do not favour the use of cephalosporins for the treatment of infections. We found evidence to promote the continued use of amoxicillin-clavulanate and aminoglycoside, gentamicin for the treatment of secondary peritonitis due to appendicitis in children. We showed that adding amoxicillin-clavulanate to the combination of metronidazole and gentamicin as initial empirical treatment provided $100 \%$ coverage of resistant organisms. The use of only amoxicillin-clavulanate and gentamicin was appropriate in $98.5 \%$ of cases.

With respect to the duration of antibiotics, the median duration of intravenous antibiotic treatment was 4 days. Patients with more severe disease required at least 5 days of intravenous antibiotics and this factor contributed to prolonged hospitalisation $>7$ days in the cohort. In the experience of the authors, the antibiotic susceptibility reports may recommend the continuation of gentamicin which is only available intravenously. We think initial treatment in the setting of appendicitis-related peritonitis using triple antibiotic combination therapy (amoxicillinclavulanate, metronidazole and gentamicin) is appropriate while awaiting definitive culture and sensitivity results. 
This may help reduce the incidence of post-operative infectious complications associated with amoxicillinresistant $E$. coli in appendicitis-related peritonitis. In addition, other factors such as attention to basic infection control strategies, the surgeon's experience and technique, the duration of the procedure, hospital and operating room environment, instrument sterilisation techniques, preoperative preparation and management of any underlying medical condition of the patient should also be considered [24]. Antibiotic treatment should of course be narrowed once sensitivity results become available. In this study, 13/69 (19\%) patients had de-escalation of treatment following laboratory susceptibility results.

Retrospective, single-centre studies may limit generalisations. Further, susceptibility to cephalosporins was not routinely available due to local antimicrobial management policy and clinicians did not adhere to a strict antimicrobial protocol. However, the study findings are in line with previously documented work in this area. We feel such findings may help in the formation of consensus guidelines/ design of future trials. Patients in this study were screened for risk factors for post-operative infection and length of stay. Independent risk factors for post-operative infection were inadequate initial empirical antibiotic treatment and the presence of amoxicillin-resistant $E$. coli. Rationale for adding other empirical antibiotics to amoxicillin-clavulanate in the treatment of appendicitis-related peritonitis in children has been presented.

\section{Conclusion}

Perforation of the appendix inevitably leads to significant bacterial contamination and morbidity. E.coli and mixed anaerobes are the predominant organisms involved in the resulting peritonitis. No single antimicrobial treatment is effective and antibiotic resistance is common. Inadequate initial empirical antibiotic and amoxicillin-clavulanate resistant $E$. coli may contribute to increased post-operative infectious complications. Based on the clinical data presented, a triple antibiotic combination of amoxicillin-clavulanate, gentamicin and metronidazole is reasonable empiric basis for treatment of appendicitis-related peritonitis.

Acknowledgments The authors thank Mr. Paul Balfe for editorial advice and reviewing the initial manuscript. We also thank Mr. Joe Bergin for help on the laboratory testing methods.

\section{Conflict of interest None.}

Open Access This article is distributed under the terms of the Creative Commons Attribution License which permits any use, distribution, and reproduction in any medium, provided the original author(s) and the source are credited.

\section{References}

1. Dumont R, Cinotti R, Lejus C et al (2011) The microbiology of community-acquired peritonitis in children. Pediatr Infect Dis J 30:131-135. doi:10.1097/INF.0b013e3181eed7a4

2. Emil S, Duong S (2007) Antibiotic therapy and interval appendectomy for perforated appendicitis in children: a selective approach. Am Surg 73:917-922

3. Kaplan S (1998) Antibiotic usage in appendicitis in children. Pediatr Infect Dis J 17:1047-1048

4. Chen CY, Chen YC, Pu HN et al (2012) Bacteriology of acute appendicitis and its implication for the use of prophylactic antibiotics. Surg Infect Larchmt. doi:10.1089/sur.2011.135

5. Rabasseda X (2010) A report from the 20th European Congress of clinical microbiology and infectious diseases (April 10-13, 2010, Vienna, Austria). Drugs Today Barc 46:533-542. doi:10. 1358/dot.2010.46.7.1519208

6. Decousser JW, Lamy B, Pina P et al (2010) Trends in antibiotic susceptibility of bloodstream pathogens in hospitalized patients in France, 1996 to 2007. Diagn Microbiol Infect Dis 66:292-300. doi:10.1016/j.diagmicrobio.2009.10.007

7. Schmitt F, Clermidi P, Dorsi M et al (2012) Bacterial studies of complicated appendicitis over a 20-year period and their impact on empirical antibiotic treatment. J Pediatr Surg 47:2055-2062. doi:10.1016/j.jpedsurg.2012.04.025

8. Brook I (1980) Bacterial studies of peritoneal cavity and postoperative surgical wound drainage following perforated appendix in children. Ann Surg 192:208-212

9. Lin WJ, Lo WT, Chu CC et al (2006) Bacteriology and antibiotic susceptibility of community-acquired intra-abdominal infection in children. J Microbiol Immunol Infect 39:249-254

10. Chan KW, Lee KH, Mou JW et al (2010) Evidence-based adjustment of antibiotic in pediatric complicated appendicitis in the era of antibiotic resistance. Pediatr Surg Int 26:157-160. doi:10.1007/s00383-009-2540-6

11. Davies HO, Alkhamesi NA, Dawson PM (2010) Peritoneal fluid culture in appendicitis: review in changing times. Int J Surg 8:426-429. doi:S1743-9191(10)00114-710.1016/j.ijsu.2010.06. 016

12. van Wijck K, de Jong JR, van Heurn LW et al (2010) Prolonged antibiotic treatment does not prevent intra-abdominal abscesses in perforated appendicitis. World J Surg 34:3049-3053. doi:10. 1007/s00268-010-0767-y

13. Adibe OO, Barnaby K, Dobies J et al (2008) Postoperative antibiotic therapy for children with perforated appendicitis: long course of intravenous antibiotics versus early conversion to an oral regimen. Am J Surg 195:141-143. doi:10.1016/j.amjsurg. 2007.10.002

14. Emil S, Laberge JM, Mikhail P et al (2003) Appendicitis in children: a ten-year update of therapeutic recommendations. J Pediatr Surg 38:236-242. doi:10.1053/jpsu.2003.50052

15. Neilson IR, Laberge JM, Nguyen LT et al (1990) Appendicitis in children: current therapeutic recommendations. J Pediatr Surg 25:1113-1116

16. Guillet-Caruba C, Cheikhelard A, Guillet M et al (2011) Bacteriologic epidemiology and empirical treatment of pediatric complicated appendicitis. Diagn Microbiol Infect Dis 69:376-381. doi:10.1016/j.diagmicrobio.2010.11.003

17. St Peter SD, Tsao K, Spilde TL et al (2008) Single daily dosing ceftriaxone and metronidazole vs standard triple antibiotic regimen for perforated appendicitis in children: a prospective randomized trial. J Pediatr Surg 43:981-985. doi:10.1016/j.jpedsurg. 2008.02.018

18. Solomkin JS, Mazuski JE, Bradley JS et al (2010) Diagnosis and management of complicated intra-abdominal infection in adults 
and children: guidelines by the Surgical Infection Society and the Infectious Diseases Society of America. Surg Infect Larchmt 11:79-109. doi:10.1089/sur.2009.9930

19. Nadler EP, Reblock KK, Ford HR et al (2003) Monotherapy versus multi-drug therapy for the treatment of perforated appendicitis in children. Surg Infect Larchmt 4:327-333. doi:10. 1089/109629603322761382

20. Bennion RS, Baron EJ, Thompson JE Jr et al (1990) The bacteriology of gangrenous and perforated appendicitis-revisited. Ann Surg 211:165-171

21. Krobot K, Yin D, Zhang Q et al (2004) Effect of inappropriate initial empiric antibiotic therapy on outcome of patients with community-acquired intra-abdominal infections requiring surgery. Eur J Clin Microbiol Infect Dis 23:682-687. doi:10. 1007/s10096-004-1199-0

22. Yellin AE, Heseltine PN, Berne TV et al (1985) The role of Pseudomonas species in patients treated with ampicillin and Sulbactam for gangrenous and perforated appendicitis. Surg Gynecol Obstet 161:303-307

23. Aronoff SC, Olson MM, Gauderer MW et al (1987) Pseudomonas aeruginosa as a primary pathogen in children with bacterial peritonitis. J Pediatr Surg 22:861-864

24. Bratzler DW, Dellinger EP, Olsen KM et al (2013) Clinical practice guidelines for antimicrobial prophylaxis in surgery. Surg Infect Larchmt 14:73-156. doi:10.1089/sur.2013.9999 